\title{
Aberrant expression of Golgi protein 73 is indicative of a poor outcome in hepatocellular carcinoma
}

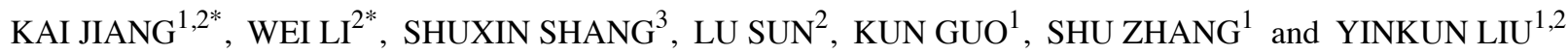 \\ ${ }^{1}$ Liver Cancer Institute, Zhongshan Hospital, Fudan University, Key Laboratory of Carcinogenesis and Cancer Invasion, \\ Ministry of Education, Shanghai 200032; ${ }^{2}$ Institutes of Biomedical Sciences, Fudan University, Shanghai 200032; \\ ${ }^{3}$ Department of Clinical Laboratory, The First Affiliated Hospital of Guangxi \\ Medical University, Nanning, Guangxi 530021, P.R. China
}

Received November 10, 2015; Accepted December 17, 2015

DOI: $10.3892 /$ or.2016.4601

\begin{abstract}
Golgi protein 73 (GP73), a resident Golgi type-II membrane protein, is often upregulated in hepatocytes. In the present study, shRNA-mediated suppression of GP73 expression in hepatocellular carcinoma (HCC) cell lines (MHCC97H, HCCLM3) resulted in a significant inhibition of cell motility and invasion and also led to the regression of epithelial-mesenchymal transition phenotypes. In contrast, overexpression of GP73 in the SMMC7721 cell line retrieved the expression of EMT markers, and promoted cell motility and invasion. High expression of GP73 was also found in HCC tissues with metastasis, as detected by western blot and immunohistochemistry analyses. Kaplan-Meier survival analysis showed that the survival of patients with high GP73 expression was significantly poorer than that of patients with low GP73 expression ( $\mathrm{p}=0.027)$. Our findings demonstrated an important role of GP73 in HCC metastasis, and indicated that GP73 is a candidate target for HCC therapy.
\end{abstract}

\section{Introduction}

HCC is the fifth most common cancer and the third leading cause of cancer-related mortality globally $(1,2)$. The 5-year survival rate of $\mathrm{HCC}$ patients is poor due to tumor recurrence and metastasis (3). The high incidence of metastasis continues to be the main obstacle of the treatment efficacy

Correspondence to: Dr Shu Zhang, Liver Cancer Institute, Zhongshan Hospital, Fudan University, 136 Yi Xue Yuan Road, Shanghai 200032, P.R. China

E-mail: zhang.shu@zs-hospital.sh.cn

*Contributed equally

Abbreviations: GP73, Golgi protein 73; HCC, hepatocellular carcinoma; EMT, epithelial-mesenchymal transition; LC, liver cirrhosis; AFP, $\alpha$-fetoprotein

Key words: invasion, metastasis, GP73, hepatocellular carcinoma, epithelial-mesenchymal transition of HCC. However, the molecular mechanisms of hepatocarcinogenesis and metastasis remain unclear. Elucidation of the detailed mechanisms of HCC cell growth and metastasis is crucial to improve HCC therapeutic intervention. Epithelialmesenchymal transition (EMT), usually occurring in the critical phases of embryonic development, is the differentiation switch from adherent epithelial cells into contractile and motile mesenchymal cells $(4,5)$. Currently, EMT of cancer cells is thought to attribute much to cancer invasion and metastasis, making it a hallmark of tumor progression (6). Although the molecular mechanism underlying tumor metastasis is still not well elucidated, investigations into this process have led to the hypothesis that many molecules are involved in EMT and play pivotal roles in tumor invasion and metastasis (7).

GP73 is a resident Golgi-specific membrane protein which is highly expressed in HCC patients. Actually, in viral and non-viral liver diseases, expression of GP73 was found to be obviously upregulated in hepatocytes (8-10). In addition, there are reports describing how GP73 is secreted to serum (11). Recent studies suggest that GP73 is a reliable biomarker for the early diagnosis of $\mathrm{HCC}$, and the sensitivity and specificity may be superior to currently used biomarker $\alpha$ fetoprotein (AFP) (12-14). Yet, this remains controversial, as other studies found that serum GP73 levels in patients with liver cirrhosis (LC) were significantly higher than those in patients with HCC; having a lower diagnostic value for $\operatorname{HCC}(15,16)$. The decreased survival and severe epithelial abnormalities in the liver and kidneys of a GP73 C-terminal truncated transgenic mouse model helped to determine the physical role of GP73 in epithelial cell function in these organs (17). Sun et al showed that an elevated level of GP73 protein is strongly associated with augmented tumor invasion and metastasis, while the exact mechanism of elevated GP73 and tumor metastasis remains largely unknown (18). GP73 was also found to be negatively correlated with E-cadherin and positively correlated with vimentin in tissues, thus it may be associated with EMT in HCC (19).

In the present study, we demonstrated that GP73 enhanced HCC cell invasion by inducing EMT and this may promote the metastasis of HCC. High expression of GP73 was also found in HCC tissues with metastasis. Kaplan-Meier survival analysis showed that the survival of patients with high GP73 
expression was significantly poorer than the survival of those with low GP73 expression, indicating it is a candidate target for HCC therapy.

\section{Materials and methods}

Cell culture. Immortalized normal human liver cell line L02 and HCC cell lines (Hep3B, HepG2, Huh7, SMMC7721, MHCC97L, MHCC97H, HCCLM3) were used in our study. L02, Hep3B, HepG2, SMMC7721 and Huh7 cells were purchased from the Chinese Academy of Sciences (Shanghai, China). MHCC97L, MHCC97H and HCCLM3 cells were established in the Liver Cancer Institute, Zhongshan Hospital. The cells were all cultured in Dulbecco's modified Eagle's medium (DMEM) supplemented with $10 \%$ fetal bovine serum (FBS) at $37^{\circ} \mathrm{C}$ with $5 \% \mathrm{CO}_{2}$.

Lentivirus construction and transfection. The GP73-RNA interference lentiviral vector was constructed by GeneChem Co., Ltd. (Shanghai, China). The double-stranded oligonucleotides targeted to GP73 mRNA targeting coding sequence (5'-AGGGAATGACAGAAACATA-3') was annealed and inserted into the shRNA expression vector pGV115-GFP. The cDNA encoding GP73 was amplified by reverse transcription polymerase chain reaction (RT-PCR) and cloned into the pGV218-GFP vector (the cells stably expressed GP73 shRNA/ GP73 proteins). Cells only expressing vectors (Mock cells) were used for the negative control. The lentivirus was generated and harvested (Shanghai GeneChem Co., Ltd.). Then, the lentivirus was transfected into targeted cells with a multiplicity of infection (MOI) of 10 to 50 (optimal MOI is 20).

Transwell migration and invasion assays. Cell migration and invasion assays were performed using a 24-well Transwell (8.0- $\mu \mathrm{m}$ pore size; Millipore, USA) precoated without or with Matrigel (BD Biosciences, USA). SMMC7721, MHCC97H or HCCLM3 cells $\left(5 \times 10^{4}\right)$ were suspended in $1.5 \mathrm{ml}$ serum-free DMEM and transferred into the inside chamber of a 24-well cell culture insert with a $8.0-\mu \mathrm{m}$ pore size. An amount of $600 \mu \mathrm{l}$ media with $20 \%$ FBS was added into the outside well. After incubation for $24 \mathrm{~h}$, the cells remaining on the upper side of the filters were cleaned with cotton-tipped swabs. Cells on the lower surface of the membrane were fixed with methanol and subjected to Giemsa staining. The cells on the underside of the filters were counted in five randomly selected fields (at x200 magnification), and the average cell number per view was calculated. All experiments were performed in triplicate.

Western blot analysis. Twenty-two HCC tissues and their paired adjacent non-tumor tissues were collected from patients undergoing resection at the Liver Cancer Institute, Zhongshan Hospital. The tissues were used for western blot analysis. General characteristics regarding these $22 \mathrm{HCC}$ patients are described in Table I. The study was approved by the Research Ethics Committee of Zhongshan Hospital, and informed consent was obtained from each patient.

Protein concentrations were determined using the bicinchoninic acid (BCA) method. Aliquots $(20 \mu \mathrm{g})$ of proteins were loaded and resolved by $10 \%$ SDS-PAGE and transferred to PVDF membranes using a Bio-Rad SemiDry apparatus. After
Table I. General characteristics of the HCC patients whose tissues were used for western blot analysis.

\begin{tabular}{lc}
\hline Characteristics & HCC $^{\mathrm{c}}$ \\
\hline No. of individuals & 22 \\
Gender [male n (\%)/female n (\%)] & $16(72.7 \%) / 6(27.3 \%)$ \\
Mean age (years) & $52 \pm 11$ \\
Edmondson-Steiner grade & $\mathrm{I}(\mathrm{n}=10), \mathrm{II}(\mathrm{n}=12)$ \\
HBV DNA (copy) $)^{\mathrm{a}}$ [mean (range)] & $2.3 \times 10^{4}\left(1.3 \times 10^{3}-1.7 \times 10^{5}\right)$ \\
AFP (ng/ml) $[$ mean (range)] & $12,354.6(3.2-70,321)$ \\
HbsAg $(\%)$ & 100 \\
AST (U/l) [mean (range)] & $130.4(16-1,630)$ \\
ALT (U/l) [mean (range)] & $110.3(14-1,120)$
\end{tabular}

${ }^{\text {a}} \mathrm{HBV}$ DNA was detected using fluorescent quantitative PCR (FQ-PCR) and had a detection limit of sensitivity of $\sim 1 \times 10^{3}$ genome equivalents per ml. ${ }^{b}$ AFP ( $\alpha$-fetoprotein) was determined using standard kits (Abbott Labs) and $20 \mathrm{ng} / \mathrm{ml}$ was considered the upper limit of the normal. ${ }^{\mathrm{C}} \mathrm{HCC}$ diagnosis was confirmed by ultrasound imaging and biopsy. AST, aspartate aminotransferase; ALT, alanine aminotransferase.

Table II. General characteristics of the HCC patient whose tissues were used for the immunohistochemical tissue microarray.

\begin{tabular}{lc}
\hline Characteristics & HCC $^{\mathrm{c}}$ \\
\hline No. of individuals & 138 \\
Gender [male n (\%)/female n (\%)] & $103(74.6 \%) / 35(25.4 \%)$ \\
Mean age (years) & $55 \pm 13$ \\
Edmondson-Steiner grade & $\mathrm{I}(\mathrm{n}=78), \mathrm{II}(\mathrm{n}=60)$ \\
HBV DNA (copy) $)^{\mathrm{a}}$ [mean (range)] & $1.8 \times 10^{4}\left(1.0 \times 10^{3}-1.3 \times 10^{5}\right)$ \\
AFP (ng/ml) ${ }^{\mathrm{b}}$ [mean (range)] & $10,345.6(3.2-70,321)$ \\
HbsAg $(\%)$ & 100 \\
AST (U/l) [mean (range)] & $134.1(18-1,567)$ \\
ALT (U/l) [mean (range)] & $120.7(16-1,256)$
\end{tabular}

${ }^{\mathrm{a}} \mathrm{HBV}$ DNA was detected with fluorescent quantitative PCR (FQ-PCR) and has a detection limit of sensitivity of $\sim 1 \times 10^{3}$ genome equivalents per $\mathrm{ml}$; ${ }^{b} \mathrm{AFP}$ ( $\alpha$-fetoprotein) was determined using standard kits and $20 \mathrm{ng} / \mathrm{ml}$ was considered the upper limit of the normal; ${ }^{\mathrm{c}} \mathrm{HCC}$ diagnosis was confirmed by ultrasound imaging and biopsy. AST, aspartate aminotransferase; ALT, alanine aminotransferase.

being blocked for non-specific binding sites, the membranes were incubated with the indicated primary antibodies: antiGP73 (1:200 dilution; Santa Cruz Biotechnology, Santa Cruz, CA, USA), E-cadherin (1:200 dilution; Abcam, Hong Kong), $\mathrm{N}$-cadherin (1:100 dilution; Invitrogen, Carlsbad, CA, USA) and GAPDH (1:10,000 dilution; Kang-Cheng, Shanghai, China) overnight at $4{ }^{\circ} \mathrm{C}$, followed by HRP-conjugated secondary antibodies for $1 \mathrm{~h}$ at room temperature. After washing three times in Tris-buffered saline with $0.1 \%$ Tween-20 (TBST), immunoreactive protein bands were visualized using an enhanced chemiluminescence (ECL) detection system (GE Healthcare, Piscataway, NJ, USA). 
A
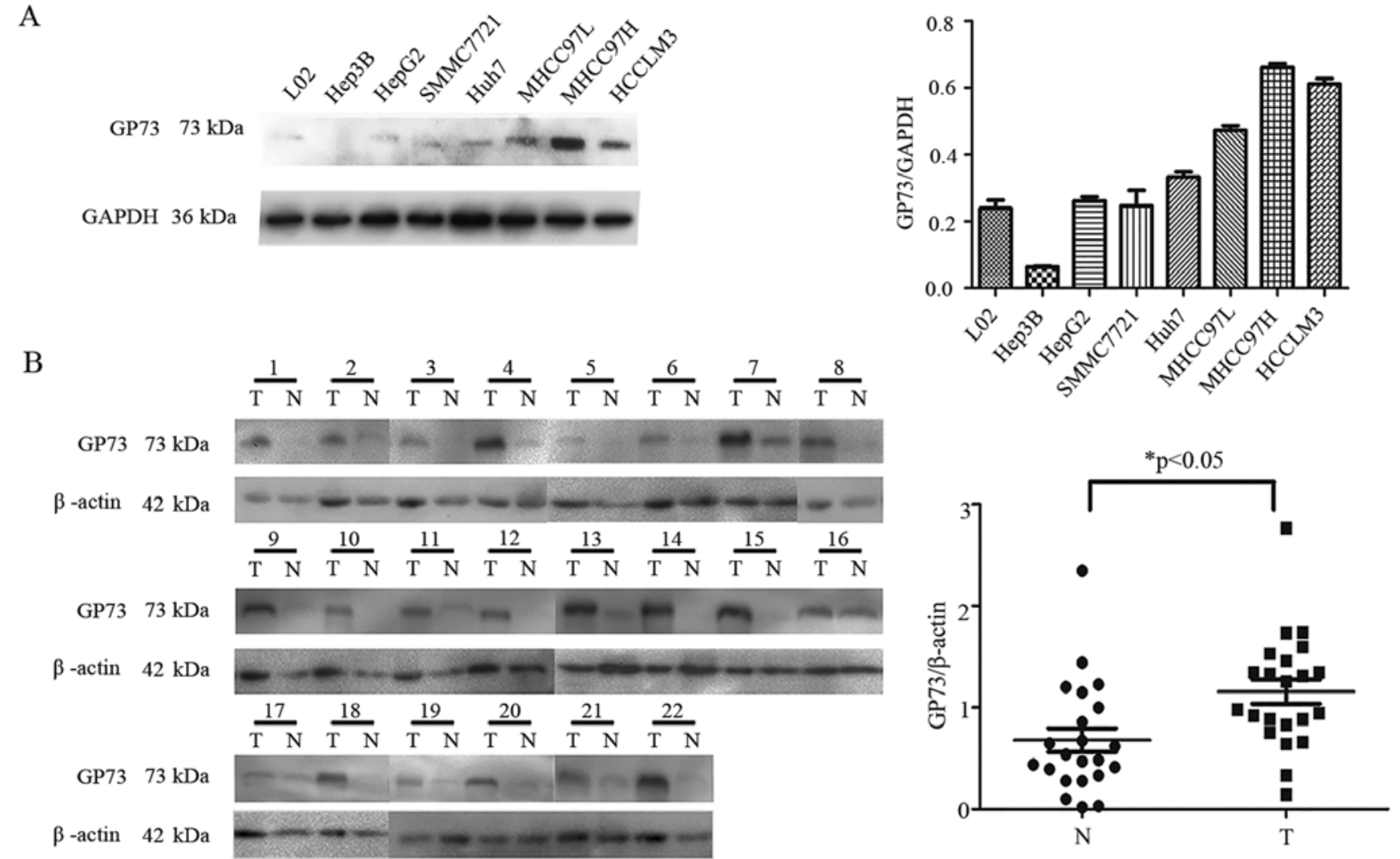

Figure 1. Expression of GP73 is upregulated in human HCCs. (A) Expression of GP73 in a human normal liver cell line (L02) and seven HCC cell lines was examined by western blot assay. (B) Representative western blotting showing the expression of GP73 in tumor tissues (T) and paired non-cancerous tissues (N) from 22 HCC patients. Compared with the paired non-cancerous tissues, the expression of GP73 in the tumor tissues was significantly increased ("p $<0.05)$.

Cell immunofluorescence assay. For immunofluorescence staining, cells grown on glass coverslip were fixed in $4 \%$ paraformaldehyde and permeabilized using $0.5 \%$ Triton X-100. Non-specific binding sites were blocked with normal goat or rabbit serum. The cells were then incubated with the primary antibodies against GP73 (1:50 dilution), E-cadherin (1:100 dilution), $\mathrm{N}$-cadherin (1:100 dilution) overnight at $4^{\circ} \mathrm{C}$. After thorough washing, the cells were then incubated with Alexa-Fluor 555 anti-mouse IgG (1:1,000 dilution; Cell Signaling Technology, Danvers, MA, USA) or anti-goat IgG (1:1,000 dilution; Abcam). Finally, the cells were washed and stained with DAPI. Images were captured using a Leica fluorescence microscope.

Immunohistochemical analysis of tissue microarrays. Tissue samples of $48 \mathrm{HCC}$ patients with or without metastasis were obtained from the Department of Hepatobiliary Surgery, The First Affiliated Hospital of Guangxi Medical University (Nanning, China). HCC diagnosis was based on World Health Organization criteria. Ethical approval was obtained from the Research Ethics Committee of The First Affiliated Hospital of Guangxi Medical University, and written informed consent was obtained from each patient. Tissue microarrays were constructed using formalin-fixed, paraffin-embedded tissue samples. Primary antibody against GP73 (1:50 dilution) and donkey anti-goat secondary antibody was used for immunohistochemical staining. Then, the integrated optical density of the tissue microarray derived from $48 \mathrm{HCC}$ patients was evaluated by IPP software (Image-Pro Plus 5.1).
Staining for GP73 in the tissue microarray of $90 \mathrm{HCC}$ patients was assessed using a previously described scoring method (20). The 90 HCC samples were obtained from the Liver Cancer Institute, Zhongshan Hospital. Ethical approval was obtained from the Research Ethics Committee of Zhongshan Hospital, and written informed consent was obtained from each patient. The staining intensity was scored on a scale of 0 to 3 as negative, weak, medium and strong, respectively. The stained area, which was calculated as the percentage of positively stained cells relative to the total cells, was scored on a scale of 0 to 4 : $0(0 \%), 1(1-25 \%), 2$ (26-50\%), $3(51-75 \%)$ and $4(76-100 \%)$. The overall score was calculated by multiplying the intensity score and the staining area score. Samples were categorized into four grades: an overall score equal to 0 was graded as '-'; an overall score equal to $1,2,3$ or 4 was graded as 't'; an overall score equal to 5, 6, 7 or 8 was graded as '++'; an overall score equal to $9,10,11$ or 12 was graded as ' +++ '. The stained tissue sections were analyzed by two pathologists without any knowledge regarding the patient clinical information. Based on the immunohistochemical grades, the patients were divided into two groups: the high expression group, which included patients with grades ' ++ ' or ' +++ ', and the low expression group, including patients graded as '-' or ' + '. General characteristics of these HCC patients for the tissue microarray are described in Table II.

Statistical analysis. Statistical analysis was performed with SPSS 15.0 for Windows (SPSS, Chicago, IL, USA). Data are presented as the mean \pm SD unless otherwise indicated. The 
A
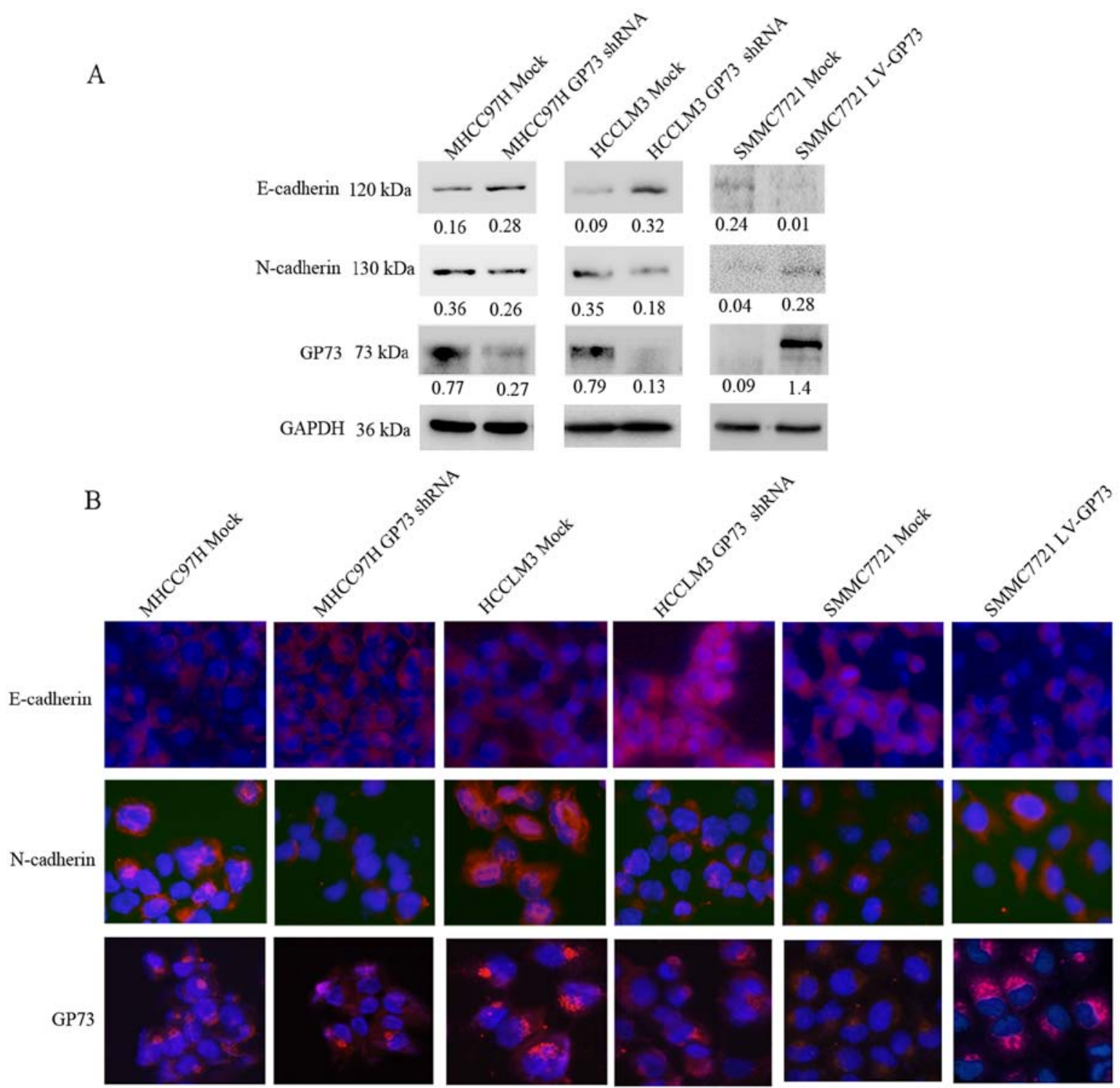

Figure 2. GP73 is correlated with EMT. (A) Expression levels of an epithelial marker (E-cadherin) and a mesenchymal marker (N-cadherin) were determined by western blot analysis in GP73-depleted MHCC97H and HCCLM3 cell lines and the GP73-overexpressing SMMC7721 cell line. (B) The expression of an epithelial marker and a mesenchymal marker in relative cell lines was detected by immunofluorescence staining (magnification, x200). Knockdown of GP73 expression may disrupt the EMT process in MHCC97H and HCCLM3 cells. Overexpression of GP73 induced EMT in the SMMC7721 cells. The ratio values were calculated using Quantity One software.

Student's t-test (two-tailed) was used to compare two groups of parametric variants, and Spearman's Rho test or Chi-square test was used to analyze non-parametric variants. $p<0.05$ was considered to indicate a statistically significant result.

\section{Results}

GP73 is correlated with cellular EMT. Expression levels of GP73 in various human cell lines (L02, Hep3B, HepG2, Huh7, SMMC7721, MHCC97L, MHCC97H and HCCLM3) were investigated. As shown in Fig. 1A, increased expression of GP73 was observed in HCC cell lines with high metastatic potential (MHCC97L, MHCC97H, HCCLM3) compared with the levels in the low or non-metastatic cell lines (L02, Hep3B, HepG2, Huh7, SMMC7721). To elucidate the effects of GP73 knockdown and overexpression on HCC cell behavior, lentiviral-mediated shRNA was used to knock down the expression of GP73 in the MHCC97H and HCCLM3 cells, while a GP73 cDNA expression vector was introduced into the SMMC7721 cells for overexpression of GP73. GP73 expression in the transfected cells was confirmed by western blotting (Fig. 2A). To examine whether GP73 promotes EMT, cellular morphology was observed. Knockdown of GP73 led to marked morphological changes from a mesenchymal-like phenotype to an epithelial-like phenotype in the MHCC97H and HCCLM3 cells (Fig. 3A and B). While in the SMMC7721 cells, overexpression of GP73 altered cells from an epitheliallike phenotype to a mesenchymal-like phenotype (Fig. 3C). Western blot and cell immunofluorescence analyses were used to detect expression of EMT markers in the GP73shRNA-treated MHCC $97 \mathrm{H}$ and HCCLM3 cells and the GP73-overexpressing SMMC7721 cells. In the GP73-shRNA- 
A

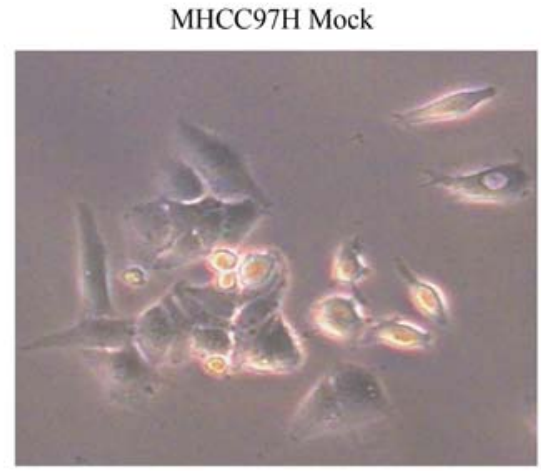

$\mathrm{B}$

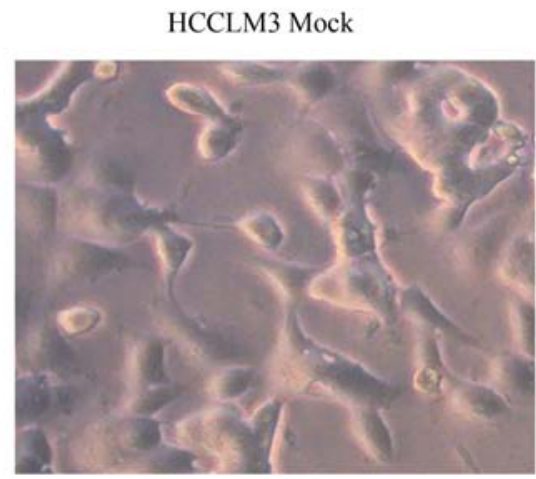

$\mathrm{C}$

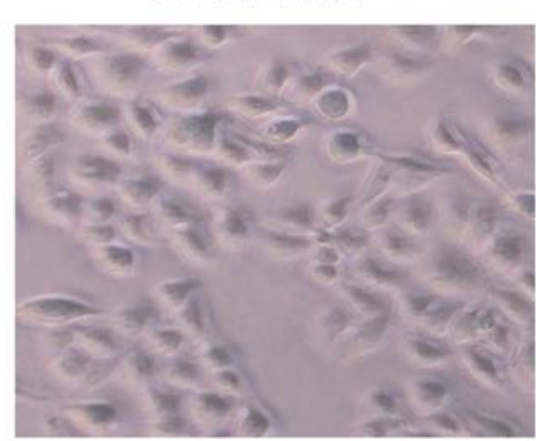

MHCC97H GP73 shRNA

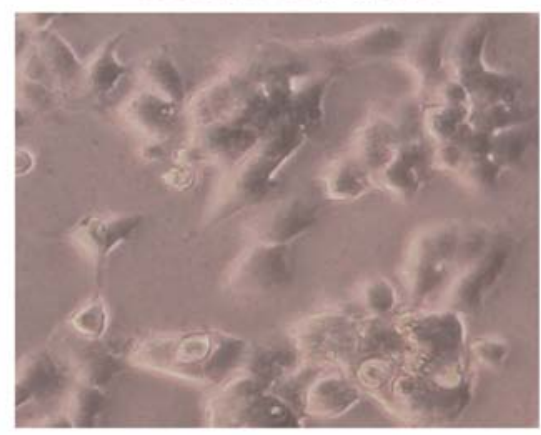

HCCLM3 GP73 shRNA

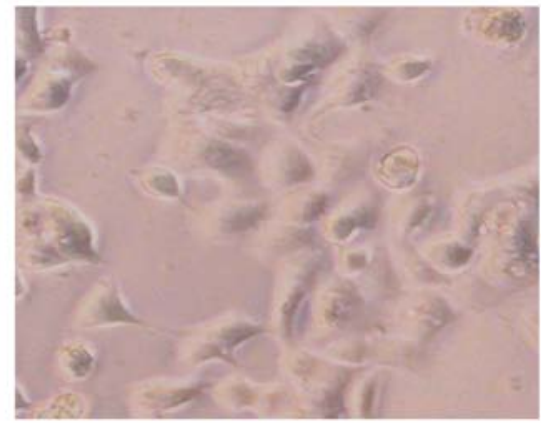

SMMC7721 LV-GP73

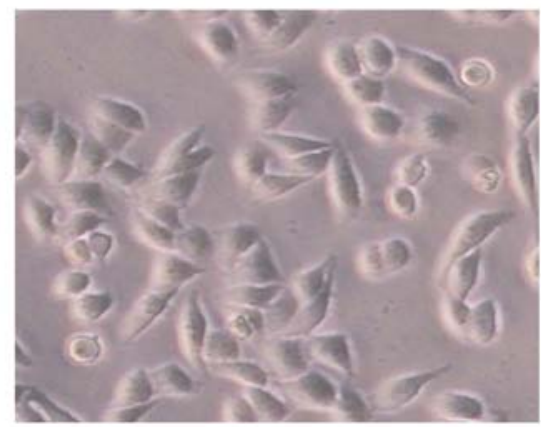

Figure 3. Morphological changes in HCC cells after knockdown and overexpression of GP73 (magnification, x200). (A) Morphological change from a mesenchymal-like phenotype to an epithelial-like phenotype in MHCC97H GP73 shRNA cells compared with MHCC97H Mock cells (B) Morphological change from a mesenchymal-like phenotype to an epithelial-like phenotype in HCCLM3 GP73 shRNA cells compared with HCCLM3 Mock cells. (C) Overexpression of GP73 altered an epithelial-like phenotype into a mesenchymal-like phenotype. [The cells stably expressed GP73 shRNA/GP73 proteins. Cells only expressing vectors (Mock cells) were used as the negative control].

treated cells, the expression of E-cadherin (an epithelial marker) was increased and the expression of $\mathrm{N}$-cadherin (a mesenchymal marker) was decreased. In contrast, the protein level of E-cadherin was downregulated and $\mathrm{N}$-cadherin was upregulated in the GP73-overexpressing cells (Fig. 2). These findings indicated that GP73 was involved in EMT in HCC cell lines.

Knockdown of GP73 expression inhibits cell motility and invasion. We also examined whether the change in GP73 expression affects the proliferation, migration and invasion of the HCC cells using the transfected (knockdown or overexpression of GP73) cell lines. MHCC97H Mock and HCCLM3 Mock cells (cells transfected with scrambled shRNA) and MHCC97H GP73 shRNA and HCCLM3 GP73
shRNA cells (cells transfected with GP73 shRNA), grew at similar rates (Fig. 4A and B). The same results (Fig. 4C) were obtained for the GP73-overexpressing SMMC7721 cells, indicating that GP73 was not required for the proliferation of HCC cells. Cell migration using a Transwell assay chamber was also assessed. Cells that migrated into the lower compartment of the migration chamber were fixed and then stained with Giemsa. Effective knockdown of GP73 in both MHCC 97H and HCCLM3 cells markedly decreased cellular motility, as the number of migrated cells was significantly less than that for the MHCC97H Mock and HCCLM3 Mock cells. The invasive activity caused by knockdown of GP73 was determined using invasion chamber assays with Matrigel. Consistent with the cell migration results, knockdown of GP73 significantly decreased cell invasion capacity compared 
A

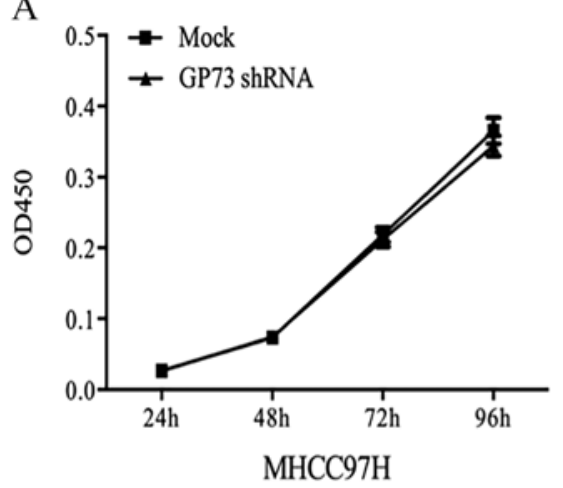

$\mathrm{C}$

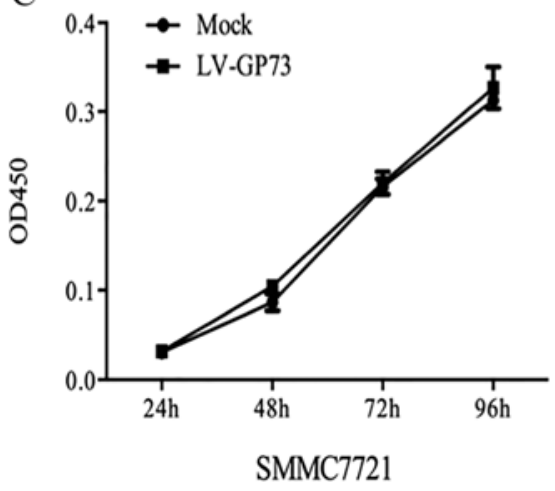

B

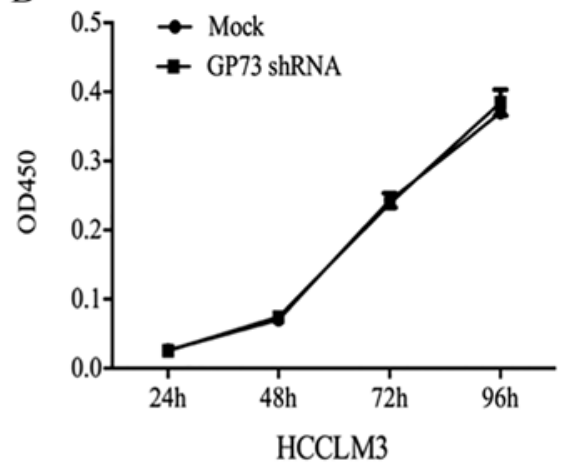

Figure 4. Suppression or overexpression of GP73 does not affect the proliferation of HCC cells in vitro. (A) MHCC97H Mock and MHCC97H GP73 shRNA cells grew at similar rates. (B) HCCLM3 Mock and HCCLM3 GP73 shRNA cells grew at similar rates. (C) SMMC7721 Mock and SMMC7721 LV-GP73 cells grew at similar rates.

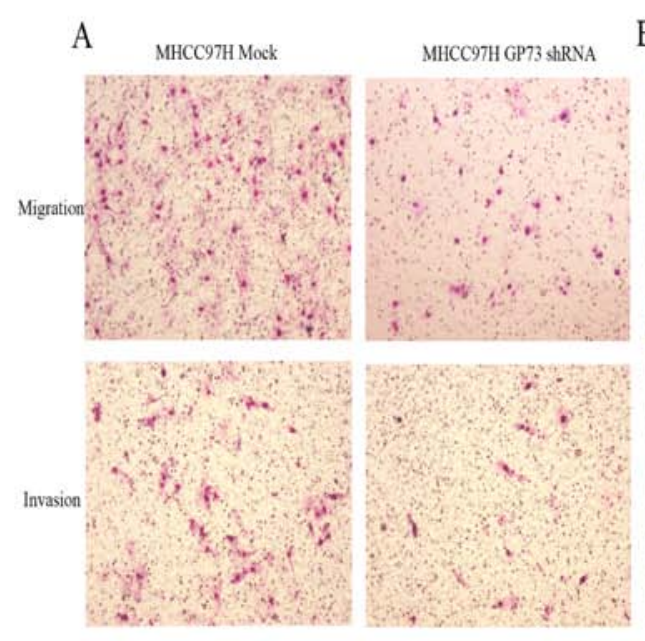

B $\quad$ HCCLM3 Mok
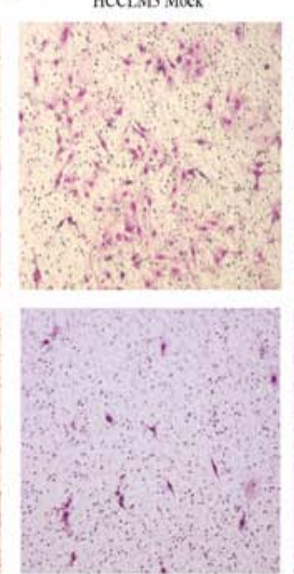

HCCLM3 GP73 shRNA

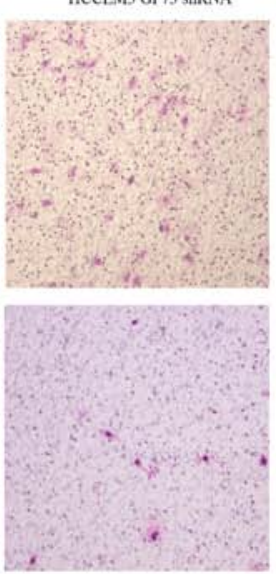

C SMMc7721 Mock

SMMC7721 LV-GP73
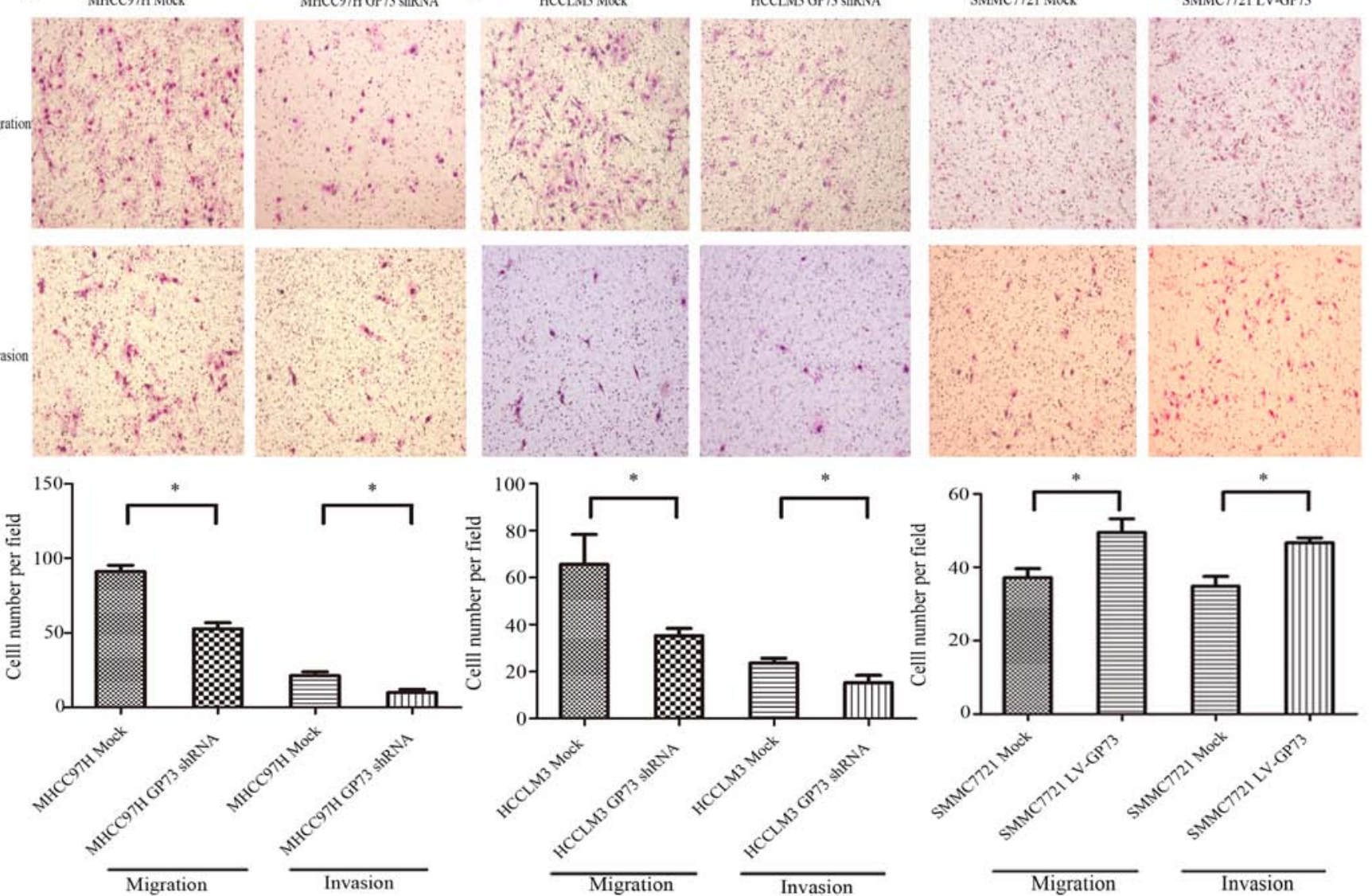

Figure 5. GP73 regulates the migration and invasion of HCC cells. The number of migratory and invasive cells in the HCC cells [(A) MHCC97H Mock vs. MHCC97H GP73 shRNA, (B) HCCLM3 Mock vs. HCCLM3 GP73 shRNA, (C) SMMC7721 Mock vs. SMMC7721 LV-GP73] as determined by migration and invasion assays. The migratory and invasive properties of the cells were analyzed as described in Materials and methods. Error bars represent SD. * $\mathrm{p}<0.05$. 
A

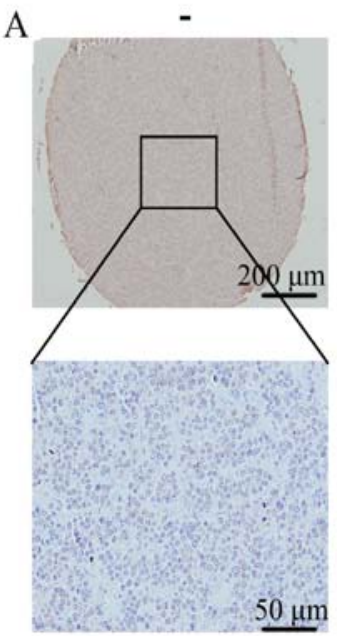

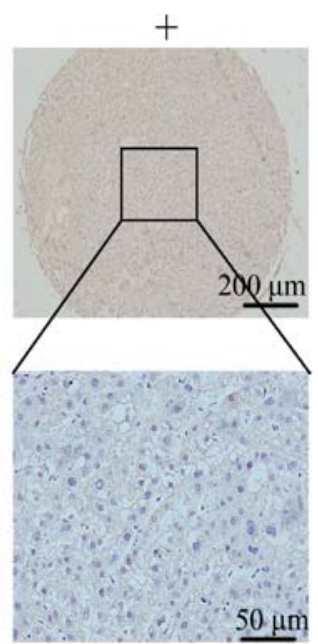

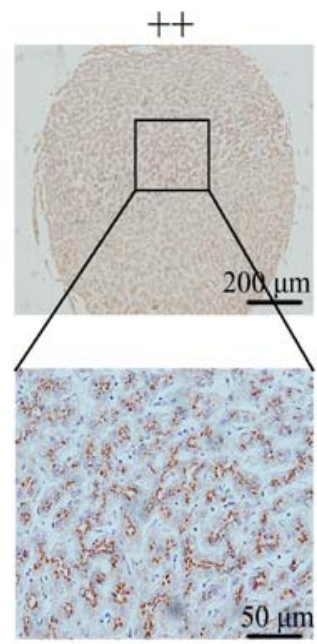

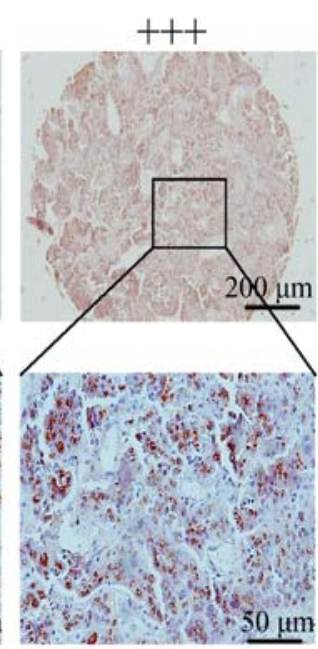

B

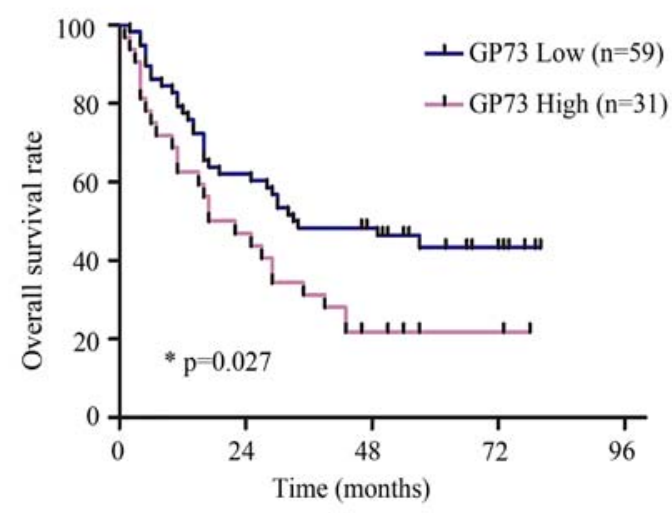

Figure 6. GP73 expression is correlated with the survival of HCC patients. (A) Representative low and high GP73 expression as shown by immunohistochemistry. (B) Kaplan-Meier analysis of overall survival in HCC patients with low GP73 expression (n=59) or high GP73 expression (n=31) using SPSS 13.0.

with the mock cells (Fig. 5A and B). Taken together, these results indicated that knockdown of GP73 in MHCC97H and HCCLM3 cells inhibited cell migratory and invasive abilities.

Overexpression of GP73 enhances cell motility and invasion. Cell migration and invasion abilities in the SMMC7721 LV-GP73 cells (cells overexpressing GP73) were assessed. The results showed that overexpression of GP73 markedly increased cell migratory and invasive abilities, as the numbers of migrated SMMC7721 LV-GP73 cells were significantly higher compared to the SMMC7721 Mock cells (cells transfected with an empty vector) (Fig. 5C). Consistent with the results of GP73 knockdown in the MHCC97H and HCCLM3 cells, GP73 overexpression in SMMC7721 cells enhanced cell invasion and metastasis.

High expression of GP73 is correlated with poor survival and metastasis of HCC patients. GP73 was significantly increased in the HCC tissues compared with the paired non-cancerous tissues in 22 patients by western blot analysis (Fig. 1B). Furthermore, HCC tissues from 90 patients with survival information from an 80-month follow-up period were collected for production of a tissue microarray. The typical images of negative and positive staining of GP73 are shown in Fig. 6A. Kaplan-Meier survival analysis showed that the survival of patients with high GP73 expression was significantly poorer than the survival of those with low GP73 expression (Fig. 6B). The GP73 level in the HCC tissues derived from the patients with metastasis was obviously increased in comparison with that in the HCC tissues from patients without metastasis by immunohistochemical analysis, indicating the possible role of GP73 in HCC metastasis and its aberrant expression was indicative of poor outcomes in HCC (Fig. 7).

\section{Discussion}

Previous studies have demonstrated the upregulation of GP73 in liver diseases $(21,22)$. In addition, upregulation of GP73 has been reported in Alzheimer's disease (23), Wilson's disease (24), prostate cancer (25-27), renal cell cancer (28) and lung cancer (29). A number of studies show that serum GP73 levels in patients with liver disease are markedly upregulated $(13,14,30)$, suggesting that GP73 may play an important role in liver disease. Hu et al showed that the transmembrane domain with a positively charged residue in the cytoplasmic N-terminal tail was necessary to support its Golgi localization (31). However, the function of GP73 remains unclear.

A previous study showed that expression of GP73 may be associated with enhanced tumor invasion and metastasis (18). In contrast, it was reported that GP73 expression had no 
A

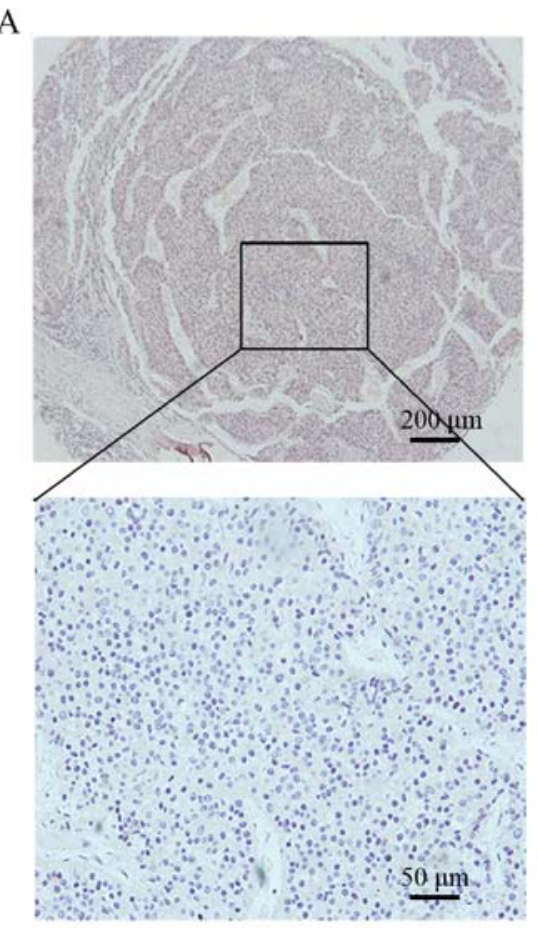

non-metastatic $\mathrm{HCC}$

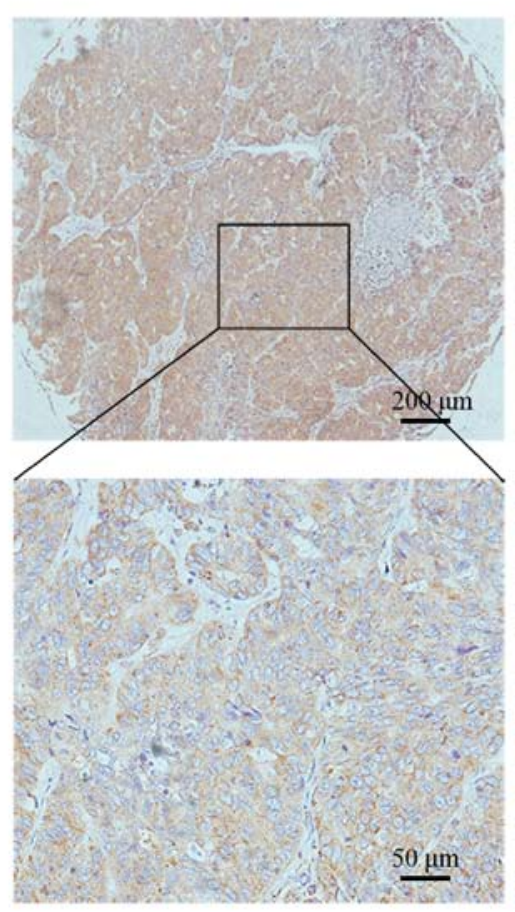

metastatic HCC

B

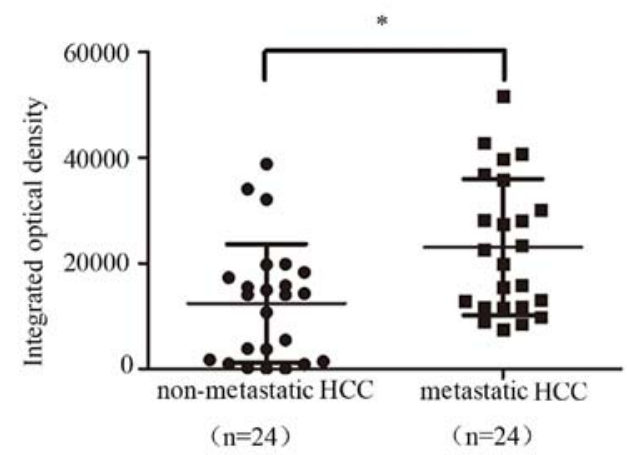

Figure 7. Immunohistochemical analysis of GP73 was performed using a tissue microarray containing 48 HCC tissues (24 non-metastatic HCC tissue dots and 24 metastatic HCC tissue dots). (A) Tissues were stained with goat anti-GP73 antibody and visualized with a donkey anti-goat secondary antibody. (B) Compared with primary HCC, the expression of GP73 in HCC specimens with metastasis was significantly increased. The integrate optical density was evaluated by IPP software (Image-Pro Plus 5.1). ${ }^{*} \mathrm{p}<0.05$.

relation with HCC metastasis (33). Different reagents used in various studies may have led to such discrepancy (34). Whether GP73 really plays a role in HCC metastasis is of great significance. Recently, GP73 was found to be associated with EMT markers in HCC (19). EMT plays a pivotal role in tumor metastasis. Evidence suggests that EMT could give rise to carcinoma cell metastasis (35-38). Although numerous factors have been identified to participate in EMT $(4,39)$, whether GP73 promotes cancer metastasis via EMT remains unclear. To investigate whether GP73 is related with EMT in HCC progression, stable cell lines were established by recombinant lentiviruses for knockdown and overexpression of GP73. The results showed that the mesenchymal marker, $\mathrm{N}$-cadherin, was upregulated in the SMMC7721 LV-GP73 cells, whereas the epithelial marker, E-cadherin, was decreased. These results were further confirmed by immunofluorescence staining analysis of the cultured cells. Opposite results were obtained for the MHCC97H GP73 shRNA and HCCLM3 GP73 shRNA cells. A significant regression of EMT features was observed; a gain in the expression of epithelial marker, E-cadherin and a loss in the expression of mesenchymal marker, $\mathrm{N}$-cadherin. Thus, GP73 was found to be involved in EMT in the HCC cell lines.

As EMT is a process involved during cancer metastasis, cell migratory and invasive abilities were also explored. The results showed that MHCC97H GP73 shRNA and HCCLM3 GP73 shRNA cells had significantly reduced cell migration and invasion capabilities, while SMMC7721 LV-GP73 cells exhibited increased cell migratory and invasive abilities. The proliferation ability of these cells was not affected. Thus, we conclude that GP73 was responsible for HCC invasion. This finding may be in agreement with the results of Sun et al which showed that a higher GP73 expression level was detected in tumors with a larger load or stronger invasiveness, indicating that the overexpression of GP73 protein may be involved in the progression of HCC (18). These data indicated that GP73 may promote $\mathrm{HCC}$ metastasis via, at least partially, induction of EMT in HCC cell lines. However, the mechanisms of how 
GP73 influences the progression of HCC and EMT remain unclear. Since GP73 is a Golgi transmembrane protein, it is unlikely whether GP73 is directly involved in the signaling pathways inducing EMT. We suspect that GP73 interacted with other important proteins directly in the EMT pathways to play its role in the process. Related reports are largely limited. Further studies should focus on this issue in order to explain the detailed mechanism of GP73 in HCC EMT.

In the present study, GP73 expression in the HCC tissues was higher than the level in the paired non-cancerous tissues. The survival of patients with high GP73 expression was significantly poorer than the survival of those with low GP73 expression, which was in accord with a study of Chen et al (32). GP73 expression in the HCC tissues with metastasis and the primary tumors was then detected. HCC tissues with metastasis had higher GP73 expression, and thus strong expression of GP73 in tumor tissues was correlated with metastasis and poor survival. Hence, GP73 may be a potential biomarker for HCC prognosis and a candidate target for HCC therapy. However, the sample size for this study was limited and more stratified samples should be considered for further study.

In conclusion, the present data indicated that GP73 may play an important role in HCC metastasis by EMT induction. High expression of GP73 was also found in HCC tissues with metastasis, and the survival of patients with high GP73 expression was significantly poorer than the survival of those with low GP73 expression. Taken together, the function of GP73 is of potential value for understanding tumor metastasis and it is a candidate target for HCC therapy.

\section{Acknowledgements}

The present study was financially supported by the China National Key Projects for Infectious Diseases (nos. 2012ZX10002009-002, 2012ZX10002009-007 and 2012ZX10002012-002), the National High Tech Program (863 program: 2012AA020204), and the National Natural Science Foundation of China (21505022). All procedures performed in the studies involving human participants were in accordance with the ethical standards of the institutional and/or national research committee and with the 1964 Helsinki Declaration and its later amendments or comparable ethical standards.

\section{References}

1. Parkin DM, Bray F, Ferlay J and Pisani P: Global cancer statistics, 2002. CA Cancer J Clin 55: 74-108, 2005.

2. Jemal A, Bray F, Center MM, Ferlay J, Ward E and Forman D: Global cancer statistics. CA Cancer J Clin 61: 69-90, 2011.

3. El-Serag HB: Hepatocellular carcinoma. N Engl J Med 365: 1118-1127, 2011.

4. Sun C, Sun L, Jiang K, Gao DM, Kang XN, Wang C, Zhang S, Huang S, Qin X, Li Y, et al: NANOG promotes liver cancer cell invasion by inducing epithelial-mesenchymal transition through NODAL/SMAD3 signaling pathway. Int J Biochem Cell Biol 45: 1099-1108, 2013.

5. Wen W, Ding J, Sun W, Fu J, Chen Y, Wu K, Ning B, Han T, Huang L, Chen C, et al: Cyclin G1-mediated epithelial-mesenchymal transition via phosphoinositide 3-kinase/Akt signaling facilitates liver cancer progression. Hepatology 55: 1787-1798, 2012.

6. Thiery JP and Sleeman JP: Complex networks orchestrate epithelial-mesenchymal transitions. Nat Rev Mol Cell Biol 7: 131-142, 2006.
7. Polyak K and Weinberg RA: Transitions between epithelial and mesenchymal states: Acquisition of malignant and stem cell traits. Nat Rev Cancer 9: 265-273, 2009.

8. Iftikhar R, Kladney RD, Havlioglu N, Schmitt-Gräff A, Gusmirovic I, Solomon H, Luxon BA, Bacon BR and Fimmel CJ: Disease- and cell-specific expression of GP73 in human liver disease. Am J Gastroenterol 99: 1087-1095, 2004.

9. Kladney RD, Bulla GA, Guo L, Mason AL, Tollefson AE, Simon DJ, Koutoubi Z and Fimmel CJ: GP73, a novel Golgi-localized protein upregulated by viral infection. Gene 249: 53-65, 2000.

10. Kladney RD, Cui X, Bulla GA, Brunt EM and Fimmel CJ: Expression of GP73, a resident Golgi membrane protein, in viral and nonviral liver disease. Hepatology 35: 1431-1440, 2002.

11. Bachert C, Fimmel C and Linstedt AD: Endosomal trafficking and proprotein convertase cleavage of cis Golgi protein GP73 produces marker for hepatocellular carcinoma. Traffic 8: 1415-1423, 2007.

12. Giannelli $\mathrm{G}$ and Antonaci S: New frontiers in biomarkers for hepatocellular carcinoma. Dig Liver Dis 38: 854-859, 2006.

13. Marrero JA, Romano PR, Nikolaeva O, Steel L, Mehta A, Fimmel CJ, Comunale MA, D'Amelio A, Lok AS and Block TM: GP73, a resident Golgi glycoprotein, is a novel serum marker for hepatocellular carcinoma. J Hepatol 43: 1007-1012, 2005.

14. Riener MO, Stenner F, Liewen H, Soll C, Breitenstein S, Pestalozzi BC, Samaras P, Probst-Hensch N, Hellerbrand C, Müllhaupt B, et al: Golgi phosphoprotein 2 (GOLPH2) expression in liver tumors and its value as a serum marker in hepatocellular carcinomas. Hepatology 49: 1602-1609, 2009.

15. Ozkan H, Erdal H, Tutkak H, Karaeren Z, Yakut M, Yüksel O and Köklü S: Diagnostic and prognostic validity of Golgi protein 73 in hepatocellular carcinoma. Digestion 83: 83-88, 2011.

16. Tian L, Wang Y, Xu D, Gui J, Jia X, Tong H, Wen X, Dong Z and Tian Y: Serological AFP/Golgi protein 73 could be a new diagnostic parameter of hepatic diseases. Int J Cancer 129: 1923-1931, 2011.

17. Wright LM, Yong S, Picken MM, Rockey D and Fimmel CJ: Decreased survival and hepato-renal pathology in mice with C-terminally truncated GP73 (GOLPH2). Int J Clin Exp Pathol 2: 34-47, 2009.

18. Sun Y, Yang H, Mao Y, Xu H, Zhang J, Li G, Lu X, Sang X, Zhao H, Zhong S, et al: Increased Golgi protein 73 expression in hepatocellular carcinoma tissue correlates with tumor aggression but not survival. J Gastroenterol Hepatol 26: 1207-1212, 2011.

19. Bao YX, Cao Q, Yang Y, Mao R, Xiao L, Zhang H, Zhao HR and Wen $\mathrm{H}$ : Expression and prognostic significance of golgiglycoprotein73 (GP73) with epithelial-mesenchymal transition (EMT) related molecules in hepatocellular carcinoma (HCC). Diagn Pathol 8: 197, 2013.

20. Masunaga R1, Kohno H, Dhar DK, Ohno S, Shibakita M, Kinugasa S, Yoshimura H, Tachibana M, Kubota H, Nagasue N: Cyclooxygenase-2 expression correlates with tumor neovascularization and prognosis in human colorectal carcinoma patients. Clin Cancer Res 6: 4064-4068, 2000.

21. Mao Y, Yang H, Xu H, Lu X, Sang X, Du S, Zhao H, Chen W, Xu Y, Chi T, et al: Golgi protein 73 (GOLPH2) is a valuable serum marker for hepatocellular carcinoma. Gut 59: 1687-1693, 2010.

22. Wei H, Li B, Zhang R, Hao X, Huang Y, Qiao Y, Hou J, Li X and $\mathrm{Li}$ X: Serum GP73, a marker for evaluating progression in patients with chronic HBV infections. PLoS One 8: e53862, 2013.

23. Li H, Wetten S, Li L, St Jean PL, Upmanyu R, Surh L, Hosford D, Barnes MR, Briley JD, Borrie M, et al: Candidate single-nucleotide polymorphisms from a genomewide association study of Alzheimer disease. Arch Neurol 65: 45-53, 2008.

24. Wright LM, Huster D, Lutsenko S, Wrba F, Ferenci P and Fimmel CJ: Hepatocyte GP73 expression in Wilson disease. J Hepatol 51: 557-564, 2009.

25. Luo JH, Yu YP, Cieply K, Lin F, Deflavia P, Dhir R, Finkelstein S, Michalopoulos G and Becich M: Gene expression analysis of prostate cancers. Mol Carcinog 33: 25-35, 2002.

26. Laxman B, Morris DS, Yu J, Siddiqui J, Cao J, Mehra R, Lonigro RJ, Tsodikov A, Wei JT, Tomlins SA, et al: A first-generation multiplex biomarker analysis of urine for the early detection of prostate cancer. Cancer Res 68: 645-649, 2008.

27. Kristiansen G, Fritzsche FR, Wassermann K, Jäger C, Tölls A, Lein M, Stephan C, Jung K, Pilarsky C, Dietel M, et al: GOLPH2 protein expression as a novel tissue biomarker for prostate cancer: Implications for tissue-based diagnostics. Br J Cancer 99: 939-948, 2008. 
28. Fritzsche FR, Riener MO, Dietel M, Moch H, Jung K and Kristiansen G: GOLPH2 expression in renal cell cancer. BMC Urol 8: 15, 2008 .

29. Zhang F, Gu Y, Li X, Wang W, He J and Peng T: Up-regulated Golgi phosphoprotein 2 (GOLPH2) expression in lung adenocarcinoma tissue. Clin Biochem 43: 983-991, 2010.

30. Block TM, Comunale MA, Lowman M, Steel LF, Romano PR, Fimmel C, Tennant BC, London WT, Evans AA, Blumberg BS, et al: Use of targeted glycoproteomics to identify serum glycoproteins that correlate with liver cancer in woodchucks and humans. Proc Natl Acad Sci USA 102: 779-784, 2005.

31. Hu L, Li L, Xie H, Gu Y and Peng T: The Golgi localization of GOLPH2 (GP73/GOLM1) is determined by the transmembrane and cytoplamic sequences. PLoS One 6: e28207, 2011.

32. Chen MH, Jan YH, Chang PM, Chuang YJ, Yeh YC, Lei HJ, Hsiao M, Huang SF, Huang CY and Chau GY: Expression of GOLM1 correlates with prognosis in human hepatocellular carcinoma. Ann Surg Oncol 20 (Suppl 3): S616-S624, 2013

33. Shan SG, Gao YT, Xu YJ, Huang Y, Zhang Q, Zhai DK, Li JB, Wang FM, Jing X, Du Z, et al: Gradually increased Golgi protein 73 expression in the progression of benign liver diseases to precancerous lesions and hepatocellular carcinoma correlates with prognosis of patients. Hepatol Res 43: 1199-1210, 2013.
34. Schrohl AS, Holten-Andersen M, Sweep F, Schmitt M, Harbeck N, Foekens J and Brünner N; European Organisation for Research and Treatment of Cancer (EORTC) Receptor and Biomarker Group: Tumor markers: From laboratory to clinical utility. Mol Cell Proteomics 2: 378-387, 2003.

35. Thiery JP: Epithelial-mesenchymal transitions in tumour progression. Nat Rev Cancer 2: 442-454, 2002.

36. Cui B, Zhang S, Chen L, Yu J, Widhopf GF II, Fecteau JF, Rassenti LZ and Kipps TJ: Targeting ROR1 inhibits epithelial-mesenchymal transition and metastasis. Cancer Res 73: 3649-3660, 2013.

37. Kang Y and Pantel K: Tumor cell dissemination: Emerging biological insights from animal models and cancer patients. Cancer Cell 23: 573-581, 2013.

38. Zhang K, Corsa CA, Ponik SM, Prior JL, Piwnica-Worms D, Eliceiri KW, Keely PJ and Longmore GD: The collagen receptor discoidin domain receptor 2 stabilizes SNAIL1 to facilitate breast cancer metastasis. Nat Cell Biol 15: 677-687, 2013.

39. Wang C, Jiang K, Kang X, Gao D, Sun C, Li Y, Sun L, Zhang S, Liu X, Wu W, et al: Tumor-derived secretory clusterin induces epithelial-mesenchymal transition and facilitates hepatocellular carcinoma metastasis. Int J Biochem Cell Biol 44: 2308-2320, 2012. 